\title{
On limit distribution of the Riemann zeta-function
}

by

\author{
A. LAurinČIKAs and G. Misevičıus (Vilnius)
}

In honour of Professor J. Kubilius

on his 75th birthday

Let $s=\sigma+i t$ be a complex variable, and let, as usual, $\zeta(s)$ denote the Riemann zeta-function. It is well known that probabilistic methods can be applied for the examination of value-distribution of the function $\zeta(s)$, and the obtained results are usually stated as probabilistic limit theorems in the sense of weak convergence of probability measures. In recent years much attention was devoted to functional limit theorems for the Riemann zeta-function. B. Bagchi [1] proved such a limit theorem in the space of meromorphic functions with the topology of uniform convergence on compacta, and the first author of this article obtained a limit theorem in the space of continuous functions [4]. The latter paper also contains a survey on limit theorems for the Riemann zeta-function. In [7] we have presented a limit theorem with weight for $\zeta(s)$ in the space of analytic functions with the topology of uniform convergence on compacta. Let $w(t)$ be a positive function with bounded variation on $\left[T_{0}, \infty\right), T_{0}>0$, such that its variance $V_{a}^{b} w$ on $[a, b]$ satisfies the inequality $V_{a}^{b} w \leq c w(a)$ with some $c>0$ for all $b \geq a \geq T_{0}$. Moreover, let

$$
U=U(T, w)=\int_{T_{0}}^{T} w(t) d t,
$$

and suppose that $\lim _{T \rightarrow \infty} U(T, w)=\infty$. Denote by $\mathcal{B}(S)$ the class of Borel sets of the space $S$, and by $G$ some region in the complex plane $\mathbb{C}$. Let $H(G)$ stand for the space of analytic functions on $G$ with the topology of uniform

Research of the first author partially supported by grant from Lithuanian Studies and Sciences Fund. 
convergence on compacta. Define two probability measures

$$
P_{j, T, w}(A)=\frac{1}{U} \int_{T_{0}}^{T} w(\tau) I_{\{\tau: \zeta(s+i \tau) \in A\}} d \tau, \quad A \in \mathcal{B}\left(H\left(D_{j}\right)\right), j=1,2,
$$

where $D_{1}=\{s \in \mathbb{C}: 1 / 2<\sigma<1\}, D_{2}=\{s \in \mathbb{C}: \sigma>1\}$, and $I_{A}$ denotes the indicator function of the set $A$. Then in [7] the following theorem was proved.

Theorem A. There are probability measures $P_{j, w}$ on $\left(H\left(D_{j}\right), \mathcal{B}\left(H\left(D_{j}\right)\right)\right)$ such that the measures $P_{j, T, w}$ converge weakly to $P_{j, w}$ as $T \rightarrow \infty$.

It is of interest to find the explicit form of the limit measure $P_{j, w}$. In case $w(t) \equiv 1$, for this purpose, ergodic theory can be applied. Unfortunately, we do not know any ergodic theorem with weight $w(t)$. Therefore we must formulate an assertion of this theorem as a condition for the function $w(t)$. First we recall the basics of ergodic theory (see, for example, [3]).

Let $(\Omega, \mathcal{F}, \mathbb{P})$ be some probability space, and let $X(\tau, \omega), \tau \in \mathcal{T}, \omega \in \Omega$, denote a random process. Let $Y$ be the space of all finite real functions $y(\tau)$, $\tau \in \mathcal{T}$. It is known that the family of finite-dimensional distributions, i.e.

$$
\mathbb{P}\left(X\left(\tau_{1}, \omega\right)<x_{1}, \ldots, X\left(\tau_{n}, \omega\right)<x_{n}\right), \quad n=1,2, \ldots,
$$

where $\tau_{1}, \ldots, \tau_{n}$ is an arbitrary set of values of $\tau$, determines a probability measure $Q$ on $(Y, \mathcal{B}(Y))$. Note that $Q(A)=\mathbb{P}\left(A^{\prime}\right)$ for $A \in \mathcal{B}(Y)$, where $A^{\prime}=\{\omega: X(\tau, \omega) \in A\}$. Then on the probability space $(Y, \mathcal{B}(Y), Q)$ the translation transformation $g_{u}$ can be defined which maps each function $y(\tau) \in Y$ to $y(\tau-u)$.

A random process $X(\tau, \omega)$ is said to be strongly stationary if all its finitedimensional distributions are invariant with respect to the translations by $u$.

Let $A_{u}=g_{u}(A)$ for $A \in \mathcal{B}(Y)$. A set $A \in \mathcal{B}(Y)$ is called an invariant set of the process $X(\tau, \omega)$ if for each $u$ the sets $A$ and $A_{u}$ differ by a set of $Q$-measure zero. In other words, $Q\left(A \triangle A_{u}\right)=0$, where $\triangle$ denotes symmetric difference. All the invariant sets form a $\sigma$-field.

We say that a strongly stationary process $X(\tau, \omega)$ is ergodic if its $\sigma$-field of invariant sets consists only of sets having $Q$-measure equal to 0 or 1 .

For an ergodic process the following classical Birkhoff-Khinchin theorem holds. Let the process $X(\tau, \omega)$ be ergodic with $E|X(\tau, \omega)|<\infty$, and let sample paths be integrable almost surely in the Riemann sense over every finite interval. Then

$$
\lim _{T \rightarrow \infty} \frac{1}{T} \int_{0}^{T} X(\tau, \omega) d t=E X(0, \omega)
$$

almost surely. Here $E X$ denotes the mean of the random variable $X$. 
Now we suppose that the function $w(t)$ satisfies some special condition. Let the process $X(\tau, \omega)$ be the same as in the Birkhoff-Khinchin theorem. Suppose that

$$
\frac{1}{U} \int_{T_{0}}^{T} w(\tau) X(t+\tau, \omega) d \tau=E X(0, \omega)+o(1+|t|)^{\alpha}
$$

almost surely for all $t \in \mathbb{R}$ with some $\alpha>0$ as $T \rightarrow \infty$.

When $w(t) \equiv 1$ and $t=0$ the equality (2) becomes (1).

For $w(t)$ satisfying $(2)$ we are now able to indicate an explicit formula for $P_{j, w}$. Since the case $j=2$ is simpler and similar to that of $j=1$, we will deal with the case $j=1$ only.

Denote by $\gamma$ the unit circle on the complex plane, that is, $\gamma=\{s \in \mathbb{C}$ : $|s|=1\}$. Moreover, let

$$
\Omega=\prod_{p} \gamma_{p},
$$

where $\gamma_{p}=\gamma$ for all primes $p$. With the product topology and pointwise multiplication $\Omega$ is a compact topological group. It is well known that there exists a unique probability Haar measure on $(\Omega, \mathcal{B}(\Omega))$. Thus we obtain the probability space $(\Omega, \mathcal{B}(\Omega), m)$. Let $\omega(p)$ stand for the projection of $\omega \in \Omega$ to the coordinate space $\gamma_{p}$. Then, setting

$$
\omega(k)=\prod_{p^{\alpha} \| k} \omega^{\alpha}(p),
$$

where $p^{\alpha} \| k$ means that $p^{\alpha} \mid k$ but $p^{\alpha+1} \nmid k$, we obtain an extension of $\omega(p)$ to the set of all natural numbers as a completely multiplicative unimodular function.

Let $D=\{s \in \mathbb{C}: \sigma>1 / 2\}$, and set

$$
\xi(s, \omega)=\sum_{k=1}^{\infty} \frac{\omega(k)}{k^{s}},
$$

where $s \in D$ and $\omega \in \Omega$. Then it is known from [1] (see also Lemma 5.1.6 of [6]) that $\xi(s, \omega)$ is an $H(D)$-valued random element on $(\Omega, \mathcal{B}(\Omega), m)$. Moreover, for almost all $\omega \in \Omega$, the product

$$
\zeta(s, \omega)=\prod_{p}\left(1-\frac{\omega(p)}{p^{s}}\right)^{-1}
$$

is uniformly convergent on compact subsets of $D$, and

$$
\sum_{k=1}^{\infty} \frac{\omega(k)}{k^{s}}=\prod_{p}\left(1-\frac{\omega(p)}{p^{s}}\right)^{-1} .
$$


Let $P_{\zeta}$ denote the distribution of $\zeta(s, \omega)$. Moreover, let $P_{j, \zeta}$ be the restriction of $P_{\zeta}$ to $\left(H\left(D_{j}\right), \mathcal{B}\left(H\left(D_{j}\right)\right)\right), j=1,2$.

Theorem. The measures $P_{j, T, w}$ converge weakly to $P_{j, \zeta}$ as $T \rightarrow \infty$.

Thus, the limit measure $P_{j, w}$ in Theorem A is independent of the function $w(t)$ and it coincides with $P_{j, \zeta}$.

We begin the proof of the Theorem by a limit theorem for trigonometric polynomials

$$
p_{n}(s)=\sum_{k=1}^{n} \frac{a(k)}{k^{s}} .
$$

Define a probability measure on $(H(G), \mathcal{B}(H(G)))$ by

$$
P_{T, n, w}(A)=\frac{1}{U} \int_{T_{0}}^{T} w(\tau) I_{\left\{\tau: p_{n}(s+i \tau) \in A\right\}} d \tau .
$$

Lemma 1. There exists a probability measure $P_{n, w}$ on $(H(G), \mathcal{B}(H(G)))$ such that $P_{T, n, w}$ converges weakly to $P_{n, w}$ as $T \rightarrow \infty$.

P r o of. See Theorem 3 of [7].

Now let $g(k), k \in \mathbb{N}$, be a unimodular completely multiplicative function, and define

$$
p_{n}(s, g)=\sum_{k=1}^{n} \frac{a(k) g(k)}{k^{s}}
$$

and

$$
\widetilde{P}_{T, n, w}(A)=\frac{1}{U} \int_{T_{0}}^{T} w(\tau) I_{\left\{\tau: p_{n}(s+i \tau, g) \in A\right\}} d \tau, \quad A \in \mathcal{B}(H(G)) .
$$

Lemma 2. The probability measures $P_{T, n, w}$ and $\widetilde{P}_{T, n, w}$ converge weakly to the same measure as $T \rightarrow \infty$.

Proof. Let $p_{1}, \ldots, p_{r}$ be the distinct primes which divide the product $\prod_{k=1, a(k) \neq 0}^{n} k$, and let

$$
\Omega_{r}=\prod_{j=1}^{r} \gamma_{p_{j}}, \quad \gamma_{p_{j}}=\gamma, j=1, \ldots, r .
$$

Moreover, let $m_{r}$ stand for the Haar measure of $\left(\Omega_{r}, \mathcal{B}\left(\Omega_{r}\right)\right)$, and let $h$ : $\Omega_{r} \rightarrow H(G)$ be given by

$$
h\left(x_{1}, \ldots, x_{r}\right)=\sum_{k=1}^{n} \frac{a(k)}{k^{s} \prod_{p_{j}^{\alpha_{j}} \| k, j \leq r} x_{j}^{\alpha_{j}}}, \quad x_{j} \in \gamma, j=1, \ldots, r .
$$


In the proof of Lemma 1 (see [7]) it was shown that $P_{n, w}=m_{r} h^{-1}$. Define $h_{1}: \Omega_{r} \rightarrow \Omega_{r}$ by

$$
h_{1}\left(x_{1}, \ldots, x_{r}\right)=\left(x_{1} e^{-i \theta_{1}}, \ldots, x_{r} e^{-i \theta_{r}}\right),
$$

where $\theta_{j}=\arg g\left(p_{j}\right), j=1, \ldots, r$. By Lemma 1 the probability measures $P_{T, n, w}$ and $\widetilde{P}_{T, n, w}$ converge weakly to $m_{r} h^{-1}$ and $m_{r} \widetilde{h}^{-1}$ as $T \rightarrow \infty$, respectively, where the function $\widetilde{h}$ is defined in a similar manner to $h$. It is easy to see that

$$
\begin{aligned}
\widetilde{h}\left(x_{1}, \ldots, x_{r}\right) & =\sum_{k=1}^{n} \frac{a(k) \prod_{p_{j}^{\alpha_{j}} \| k, j \leq r} e^{i \alpha_{j} \theta_{j}}}{k^{s} \prod_{p_{j}^{\alpha_{j}} \| k, j \leq r} x_{j}^{\alpha_{j}}}=\sum_{k=1}^{n} \frac{a(k)}{k^{s} \prod_{p_{j}^{\alpha_{j}} \| k, j \leq r} x_{j}^{\alpha_{j}} e^{-i \alpha_{j} \theta_{j}}} \\
& =h\left(h_{1}\left(x_{1}, \ldots, x_{r}\right)\right) .
\end{aligned}
$$

Consequently,

$$
m_{r} \widetilde{h}^{-1}=m_{r}\left(h\left(h_{1}\right)\right)^{-1}=\left(m_{r} h_{1}^{-1}\right) h^{-1} .
$$

Since the Haar measure $m_{r}$ is invariant with respect to translation by points in $\Omega_{r}$, it follows from (3) that $m_{r} \widetilde{h}^{-1}=m_{r} h^{-1}$. This proves the lemma.

Now we will prove a similar assertion to Lemma 2 for absolutely convergent Dirichlet series. Let $\sigma_{1}>1 / 2$ be fixed. We define the function

$$
l_{n}(s)=\frac{s}{\sigma_{1}} \Gamma\left(\frac{s}{\sigma_{1}}\right) n^{s}, \quad n \in \mathbb{N},
$$

in the strip $-\sigma_{1} \leq \sigma \leq \sigma_{1}$. Here $\Gamma(s)$ stands for the Euler gamma-function. Suppose $\sigma>1 / 2$ and

$$
\zeta_{n}(s)=\frac{1}{2 \pi i} \int_{\sigma_{1}-i \infty}^{\sigma_{1}+i \infty} \zeta(s+z) l_{n}(z) \frac{d z}{z} .
$$

Since $\Gamma(\sigma+i t)=B e^{-c_{1}|t|}$ uniformly in $\sigma,|\sigma| \leq c_{2}$, the above integral exists. Here and afterwards $B$ denotes a number (not always the same) bounded by a constant.

Lemma 3. Let $K$ be a compact subset of the strip $D_{1}$. Then

$$
\lim _{n \rightarrow \infty} \limsup _{T \rightarrow \infty} \frac{1}{U} \int_{T_{0}}^{T} w(\tau) \sup _{s \in K}\left|\zeta(s+i \tau)-\zeta_{n}(s+i \tau)\right| d \tau=0 .
$$

Proof. This is Lemma 5 of [7], where its proof can be found. Note that the condition (2) is not used in the proof.

In the proof of Lemma 3 it has been shown that

$$
\zeta_{n}(s)=\sum_{m=1}^{\infty} \frac{1}{m^{s}} \exp \left\{-\left(\frac{m}{n}\right)^{\sigma_{1}}\right\}
$$


the series being absolutely convergent for $\sigma>1 / 2$. Moreover, for $n \in \mathbb{N}$ and $\omega \in \Omega$, let

$$
\begin{aligned}
\zeta_{n, N}(s) & =\sum_{m=1}^{N} \frac{1}{m^{s}} \exp \left\{-\left(\frac{m}{n}\right)^{\sigma_{1}}\right\}, \\
\zeta_{n, N}(s, \omega) & =\sum_{m=1}^{N} \frac{\omega(m)}{m^{s}} \exp \left\{-\left(\frac{m}{n}\right)^{\sigma_{1}}\right\}, \\
\zeta_{n}(s, \omega) & =\sum_{m=1}^{\infty} \frac{\omega(m)}{m^{s}} \exp \left\{-\left(\frac{m}{n}\right)^{\sigma_{1}}\right\},
\end{aligned}
$$

the latter series also being absolutely convergent for $\sigma>1 / 2$.

We define two probability measures on $\left(H\left(D_{1}\right), \mathcal{B}\left(H\left(D_{1}\right)\right)\right)$ :

$$
\begin{aligned}
& P_{T, n, w}^{1}(A)=\frac{1}{U} \int_{T_{0}}^{T} w(\tau) I_{\left\{\tau: \zeta_{n}(s+i \tau) \in A\right\}} d \tau, \\
& \widetilde{P}_{T, n, w}^{1}(A)=\frac{1}{U} \int_{T_{0}}^{T} w(\tau) I_{\left\{\tau: \zeta_{n}(s+i \tau, \omega) \in A\right\}} d \tau .
\end{aligned}
$$

Lemma 4. There is a probability measure $P_{n, w}^{1}$ on $\left(H\left(D_{1}\right), \mathcal{B}\left(H\left(D_{1}\right)\right)\right)$ such that both the measures $P_{T, n, w}^{1}$ and $\widetilde{P}_{T, n, w}^{1}$ converge weakly to $P_{n, w}^{1}$ as $T \rightarrow \infty$.

Proof. Define the probability measures

$$
\begin{aligned}
& P_{T, n, N, w}(A)=\frac{1}{U} \int_{T_{0}}^{T} w(\tau) I_{\left\{\tau: \zeta_{n, N}(s+i \tau) \in A\right\}} d \tau \\
& \widetilde{P}_{T, n, N, w}(A)=\frac{1}{U} \int_{T_{0}}^{T} w(\tau) I_{\left\{\tau: \zeta_{n, N}(s+i \tau, \omega) \in A\right\}} d \tau
\end{aligned}
$$

on $\left(H\left(D_{1}\right), \mathcal{B}\left(H\left(D_{1}\right)\right)\right)$. Then by Lemma 2 both $P_{T, n, N, w}$ and $\widetilde{P}_{T, n, N, w}$ converge weakly to the same measure $P_{n, N, w}$, say, as $T \rightarrow \infty$. Repeating the proof of Lemma 8 of [7], we deduce that the family $\left\{P_{n, N, w}: N \geq 1\right\}$ of probability measures is tight. Hence by the Prokhorov theorem it is relatively compact.

From the definitions of $\zeta_{n, N}(s)$ and $\zeta_{n}(s)$ we see that, for $\sigma>1 / 2$,

$$
\lim _{N \rightarrow \infty} \zeta_{n, N}(s)=\zeta_{n}(s),
$$

and since the series for $\zeta_{n}(s)$ converges absolutely for $\sigma>1 / 2$, the convergence is uniform on the half-plane $\sigma \geq 1 / 2+\varepsilon_{1}$ for every $\varepsilon_{1}>0$. Hence we 
find that for every $\varepsilon>0$,

(4) $\quad \lim _{N \rightarrow \infty} \limsup _{T \rightarrow \infty} \frac{1}{U} \int_{T_{0}}^{T} w(\tau) I_{\left\{\tau: \varrho\left(\zeta_{n, N}(s+i \tau), \zeta_{n}(s+i \tau)\right) \geq \varepsilon\right\}} d \tau$

$$
\leq \lim _{N \rightarrow \infty} \limsup _{T \rightarrow \infty} \frac{1}{U \varepsilon} \int_{T_{0}}^{T} w(\tau) \varrho\left(\zeta_{n, N}(s+i \tau), \zeta_{n}(s+i \tau)\right) d \tau=0 .
$$

Here $\varrho\left(f_{1}, f_{2}\right)$ is a metric on $H\left(D_{1}\right)$ defined by

$$
\varrho\left(f_{1}, f_{2}\right)=\sum_{n=1}^{\infty} 2^{-n} \frac{\varrho_{n}\left(f_{1}, f_{2}\right)}{1+\varrho_{n}\left(f_{1}, f_{2}\right)}
$$

where

$$
\varrho_{n}\left(f_{1}, f_{2}\right)=\sup _{s \in K_{n}}\left|f_{1}(s)-f_{2}(s)\right|, \quad f_{1}, f_{2} \in H\left(D_{1}\right),
$$

and $\left\{K_{n}\right\}$ is a sequence of compact subsets of $D_{1}$ such that $D_{1}=\bigcup_{n=1}^{\infty} K_{n}$, $K_{n} \subset K_{n+1}, n=1,2, \ldots$, and if $K$ is a compact set and $K \subset D_{1}$ then $K \subseteq K_{n}$ for some $n$.

Let $\eta$ be a random variable on $(\widetilde{\Omega}, \mathcal{F}, \mathbb{P})$ such that

$$
\mathbb{P}(\eta \in A)=\frac{1}{U} \int_{T_{0}}^{T} w(t) I_{A} d t, \quad A \in \mathcal{B}(\mathbb{R}) .
$$

We put

$$
X_{T, n, N}(s)=\zeta_{n, N}(s+i \eta), \quad X_{T, n}(s)=\zeta_{n}(s+i \eta) .
$$

Then by (4) we deduce that for every $\varepsilon>0$

$$
\lim _{N \rightarrow \infty} \limsup _{T \rightarrow \infty} \mathbb{P}\left(\varrho\left(X_{T, n, N}(s), X_{T, n}(s)\right) \geq \varepsilon\right)=0 .
$$

Since the family $\left\{P_{n, N, w}: N \geq 1\right\}$ is relatively compact, we may find a subsequence $\left\{P_{n, N^{\prime}, w}\right\}$ such that $P_{n, N^{\prime}, w}$ converges weakly to $P_{n, w}$, say, as $N^{\prime} \rightarrow \infty$. Using our notation, we can write

$$
X_{n, N} \underset{N \rightarrow \infty}{\stackrel{\mathcal{D}}{\longrightarrow}} P_{n, w}
$$

where $X_{n, N}$ stands for an $H\left(D_{1}\right)$-valued random element with distribution $P_{n, N, w}$. Since $P_{T, n, N, w}$ converges weakly to $P_{n, N, w}$ as $T \rightarrow \infty$, we have

$$
X_{T, n, N} \underset{T \rightarrow \infty}{\stackrel{\mathcal{D}}{\longrightarrow}} X_{n, N}
$$

where $P_{n, N, w}$ is the distribution of $X_{n, N}$. The space $H\left(D_{1}\right)$ is separable. Consequently, using (5)-(7) and applying Theorem 4.2 of [2], we obtain

$$
X_{T, n} \underset{T \rightarrow \infty}{\stackrel{\mathcal{D}}{\longrightarrow}} P_{n, w}
$$


Therefore, there exists a measure $P_{n, w}$ such that $P_{T, n, w}^{1}$ converges weakly to $P_{n, w}$ as $T \rightarrow \infty$. The relation (8) shows that $P_{n, w}$ is independent of the choice of the subsequence $\left\{P_{n, N^{\prime}, w}\right\}$. Since the family $\left\{P_{n, N, w}: N \geq 1\right\}$ is relatively compact, we find, by Theorem 2.3 of [2], that $P_{n, N, w}$ converges weakly to $P_{n, w}$ as $N \rightarrow \infty$, that is,

$$
X_{n, N} \underset{N \rightarrow \infty}{\stackrel{\mathcal{D}}{\longrightarrow}} P_{n, w} .
$$

Reasoning as above for the random elements

$$
\widetilde{X}_{T, n, N}(s, \omega)=\zeta_{n, N}(s+i \eta, \omega)
$$

and

$$
\widetilde{X}_{T, n}(s, \omega)=\zeta_{n}(s+i \eta, \omega),
$$

we find by (9) that $\widetilde{P}_{T, n, w}^{1}$ converges weakly to $P_{n, w}$ as $T \rightarrow \infty$. Thus, the lemma is proved.

Let $a_{\tau}=\left\{p^{-i \tau}: p \in \mathcal{P}\right\}$ for $\tau \in \mathbb{R}$, where $\mathcal{P}$ denotes the set of all prime numbers. Then $\left\{a_{\tau}: \tau \in \mathbb{R}\right\}$ is a one-parameter group. Define the one-parameter family $\left\{\varphi_{\tau}: \tau \in \mathbb{R}\right\}$ of transformations on $\Omega$ by setting $\varphi_{\tau}(\omega)=a_{\tau} \omega$ for $\omega \in \Omega$. Then $\left\{\varphi_{\tau}: \tau \in \mathbb{R}\right\}$ is a one-parameter group of measurable transformations of $\Omega$. As in the case of the process, a set $A \in \mathcal{B}(\Omega)$ is called an invariant set with respect to the group $\left\{\varphi_{\tau}: \tau \in \mathbb{R}\right\}$ if for each $\tau$ the sets $A$ and $A_{\tau}=\varphi_{\tau}(A)$ differ by a set of $m$-measure zero. In other words, $m\left(A \triangle A_{\tau}\right)=0$. All the invariant sets form a $\sigma$-field which is a sub- $\sigma$-field of $\mathcal{B}(\Omega)$.

Lemma 5. The one-parameter group $\left\{\varphi_{\tau}: \tau \in \mathbb{R}\right\}$ is ergodic, that is, its $\sigma$-field of invariant sets consists only of sets having $m$-measure equal to 0 or 1 .

Proof. This is Lemma 3.4.2 of [1] (see also Theorem 5.3.6 of [6]).

Now we apply the above mentioned elements of ergodic theory to the $H(D)$-valued random element $\xi(s, \omega)$.

Lemma 6. Let $T \rightarrow \infty$ and $\sigma>1 / 2$. Then

$$
\int_{T_{0}}^{T} w(t)|\xi(\sigma+i t+i \tau, \omega)|^{2} d \tau=B U(1+|t|)^{\alpha}
$$

for almost all $\omega \in \Omega$ and all $t \in \mathbb{R}$.

Proof. Let

$$
\xi(\sigma+i t, \omega)=\sum_{k=1}^{\infty} \xi_{k}(\sigma+i t, \omega) \quad \text { and } \quad \xi_{0}(\sigma+i t, \omega)=|\xi(\sigma+i t, \omega)|^{2},
$$


where

$$
\xi_{k}(\sigma+i t, \omega)=\frac{\omega(k)}{k^{\sigma+i t}}, \quad k \in \mathbb{N} .
$$

Taking into account the equality

$$
E\left|\xi_{k}(\sigma+i t, \cdot)\right|^{2}=1 / k^{2 \sigma}
$$

and the pairwise orthogonality of the random variables $\xi_{k}(\sigma+i t, \omega)$, we find that

$$
E \xi_{0}(\sigma+i t, \omega)=\sum_{k=1}^{\infty} E\left|\xi_{k}(\sigma+i t, \omega)\right|^{2}=\sum_{k=1}^{\infty} 1 / k^{2 \sigma}<\infty
$$

for every $t \in \mathbb{R}$. It is obvious that

$$
\xi_{0}\left(\sigma+i t, \varphi_{\tau}(\omega)\right)=\left|\xi\left(\sigma+i t, a_{\tau} \omega\right)\right|^{2}=|\xi(\sigma+i t+i \tau, \omega)|^{2}
$$

for almost all $\omega \in \Omega$. Since the Haar measure $m$ is $\varphi_{\tau}$-invariant, the equality $m\left(\varphi_{\tau}(A)\right)=m(A)$ is valid for each $A \in \mathcal{B}(\Omega)$ and every $\tau \in \mathbb{R}$. Therefore, $|\xi(\sigma+i t, \omega)|^{2}$ is a strongly stationary process. It is also ergodic. In fact, let $A$ be an invariant set of $|\xi(\sigma+i t, \omega)|^{2}$, i.e. by the notation used above

$$
Q\left(A \triangle A_{u}\right)=0,
$$

where $A_{u}=g_{u}(A)$. We have

$$
\begin{aligned}
A^{\prime} & \stackrel{\text { def }}{=}\left\{\omega \in \Omega:|\xi(\sigma+i \tau, \omega)|^{2} \in A\right\}=\left\{\omega \in \Omega:\left|\xi\left(\sigma, a_{\tau} \omega\right)\right|^{2} \in A\right\}, \\
A_{u}^{\prime} & \stackrel{\text { def }}{=}\left\{\omega \in \Omega:|\xi(\sigma+i \tau, \omega)|^{2} \in A_{u}\right\}=\left\{\omega \in \Omega:|\xi(\sigma+i \tau+i u, \omega)|^{2} \in A\right\} \\
& =\left\{\omega \in \Omega:\left|\xi\left(\sigma+i \tau, a_{u} \omega\right)\right|^{2} \in A\right\} .
\end{aligned}
$$

Therefore $A_{u}^{\prime}=\varphi_{u}\left(A^{\prime}\right)$. Moreover, $\left(A \triangle A_{u}\right)^{\prime}=A^{\prime} \triangle A_{u}^{\prime}$. From this and from (12) we deduce that

$$
m\left(A^{\prime} \triangle A_{u}^{\prime}\right)=m\left(\left(A \triangle A_{u}\right)^{\prime}\right)=Q\left(A \triangle A_{u}\right)=0,
$$

that is, $A^{\prime}$ is an invariant set with respect to $\varphi_{\tau}$. By Lemma 5 the group $\left\{\varphi_{\tau}: \tau \in \mathbb{R}\right\}$ is ergodic. Therefore $m\left(A^{\prime}\right)=0$ or $m\left(A^{\prime}\right)=1$. Hence $Q(A)=0$ or $Q(A)=1$, i.e., the process $|\xi(\sigma+i t, \omega)|^{2}$ is ergodic.

Observing that $\xi_{0}\left(\sigma, \varphi_{\tau}(\omega)\right) \geq 0$ and using (11) we deduce from (2) that

$$
\begin{aligned}
\frac{1}{U} \int_{T_{0}}^{T} w(\tau) \xi_{0}\left(\sigma+i t, \varphi_{\tau}(\omega)\right) d \tau & =\frac{1}{U} \int_{T_{0}}^{T} w(\tau)|\xi(\sigma+i t+i \tau, \omega)|^{2} d \tau \\
& =E \xi_{0}(\sigma, \cdot)+o(1+|t|)^{\alpha}
\end{aligned}
$$

for almost all $\omega \in \Omega$ and all $t \in \mathbb{R}$ as $T \rightarrow \infty$ with some $\alpha>0$. From this by (10) we obtain the assertion of the lemma. 
The series

$$
\sum_{k=1}^{\infty} \frac{\omega(k)}{k^{s}}
$$

converges uniformly on compact subsets of $D_{1}$ for almost all $\omega \in \Omega$. This is obtained in the proof of the fact that $\xi(s, \omega)$ is an $H(D)$-valued random element [1] (see also Lemma 5.1.6 of [6]). By Lemma 6,

$$
\int_{T_{0}}^{T} w(\tau)|\xi(\sigma+i t+i \tau, \omega)|^{2} d \tau=B U(1+|t|)^{\alpha}
$$

for $\sigma>1 / 2$ and also for almost all $\omega \in \Omega$. Denote by $\Omega_{1}$ the subset of $\omega \in \Omega$ such that both the latter assertions are true. Then $m\left(\Omega_{1}\right)=1$.

Lemma 7. Let $K$ be a compact subset of $D_{1}$. Then

$$
\lim _{n \rightarrow \infty} \limsup _{T \rightarrow \infty} \frac{1}{U} \int_{T_{0}}^{T} w(\tau) \sup _{s \in K}\left|\xi\left(s+i \tau, \omega_{1}\right)-\zeta_{n}\left(s+i \tau, \omega_{1}\right)\right| d \tau=0
$$

for $\omega_{1} \in \Omega$.

Pro of. Let $\sigma_{1}>1 / 2$, as above, be fixed. Then the function $\xi(s+z, \omega)$, for $\operatorname{Re} z=\sigma_{1}$ and $s \in K$, is represented by the absolutely convergent Dirichlet series

$$
\xi(s+z, \omega)=\sum_{m=1}^{\infty} \frac{\omega(m)}{m^{s+z}} .
$$

Consider the series

$$
\sum_{m=1}^{\infty} \frac{a_{n}\left(m, \omega_{1}\right)}{m^{s}}, \quad \omega_{1} \in \Omega_{1},
$$

where

$$
a_{n}\left(m, \omega_{1}\right)=\frac{1}{2 \pi i} \int_{\sigma_{1}-i \infty}^{\sigma_{1}+i \infty} \frac{\omega_{1}(m) l_{n}(s)}{s m^{s}} d s .
$$

Since

$$
a_{n}\left(m, \omega_{1}\right)=B m^{-\sigma_{1}} \int_{-\infty}^{\infty}\left|l_{n}\left(\sigma_{1}+i t\right)\right| d t=B m^{-\sigma_{1}},
$$

the series (13) converges absolutely for $\sigma>1 / 2$. Thus, interchanging sum and integral in

$$
\frac{1}{2 \pi i} \int_{\sigma_{1}-i \infty}^{\sigma_{1}+i \infty} \xi\left(s+z, \omega_{1}\right) l_{n}(z) \frac{d z}{z}
$$


we find that the latter integral is equal to

$$
\sum_{m=1}^{\infty} \frac{a_{n}\left(m, \omega_{1}\right)}{m^{s}}
$$

Taking into account the equality

$$
\frac{1}{2 \pi i} \int_{b-i \infty}^{b+i \infty} \Gamma(s) a^{-s} d s=e^{-a}
$$

which is valid for $b>0$ and $a>0$, we obtain

$$
a_{n}\left(m, \omega_{1}\right)=\omega_{1}(m) \exp \left\{-\left(\frac{m}{n}\right)^{\sigma_{1}}\right\} .
$$

Thus, for $\sigma>1 / 2$, the sum of the series (15) and also the integral (14) are $\zeta_{n}\left(s, \omega_{1}\right)$.

Now we will change the contour in the integral (14). The integrand has a simple pole at $z=0$. Let $\sigma$ belong to $\left[1 / 2+\varepsilon, 1-\varepsilon_{1}\right], \varepsilon>0, \varepsilon_{1}>0$, when $s \in K$. We put $\sigma_{2}=1 / 2+\varepsilon / 2$. Then by the residue theorem we have

$$
\zeta_{n}\left(s, \omega_{1}\right)=\frac{1}{2 \pi i} \int_{\sigma_{2}-\sigma-i \infty}^{\sigma_{2}-\sigma+i \infty} \xi\left(s+z, \omega_{1}\right) l_{n}(z) \frac{d z}{z}+\xi\left(s, \omega_{1}\right) .
$$

Let $L$ be a simple closed contour lying in $D_{1}$ and enclosing the set $K$, and let $\delta$ denote the distance of $L$ from the set $K$. Then by the Cauchy formula we get

$$
\begin{aligned}
\sup _{s \in K} \mid \xi\left(s+i \tau, \omega_{1}\right)-\zeta_{n}(s+ & \left.i \tau, \omega_{1}\right) \mid \\
& \leq \frac{1}{2 \pi \delta} \int_{L}\left|\xi\left(z+i \tau, \omega_{1}\right)-\zeta_{n}\left(z+i \tau, \omega_{1}\right)\right||d z| .
\end{aligned}
$$

Therefore we obtain

$$
\begin{aligned}
& \frac{1}{U} \int_{T_{0}}^{T} w(\tau) \sup _{s \in K}\left|\xi\left(s+i \tau, \omega_{1}\right)-\zeta_{n}\left(s+i \tau, \omega_{1}\right)\right| d \tau \\
& =\frac{B}{U \delta} \int_{L}|d z| \int_{T_{0}+\operatorname{Im} z}^{T+\operatorname{Im} z} w(t-\operatorname{Im} z)\left|\xi\left(\operatorname{Re} z+i t, \omega_{1}\right)-\zeta_{n}\left(\operatorname{Re} z+i t, \omega_{1}\right)\right| d t \\
& =\frac{B|L|}{U \delta} \sup _{\sigma+i u \in L} \int_{T_{0}+u}^{T+u} w(t-u)\left|\xi\left(\sigma+i t, \omega_{1}\right)-\zeta_{n}\left(\sigma+i t, \omega_{1}\right)\right| d t
\end{aligned}
$$

Here $|L|$ denotes the length of the contour $L$. The contour $L$ can be chosen so that, for $s \in L$, the inequalities $\sigma \geq 1 / 2+3 \varepsilon / 4$ and $\delta \geq \varepsilon / 4$ hold. 
By (16),

$$
\xi\left(\sigma+i t, \omega_{1}\right)-\zeta_{n}\left(\sigma+i t, \omega_{1}\right)=B \int_{-\infty}^{\infty}\left|\xi\left(\sigma_{2}+i t+i \tau\right)\right|\left|l_{n}\left(\sigma_{2}-\sigma+i \tau\right)\right| d \tau
$$

Hence we find that

$$
\begin{aligned}
& \frac{1}{U} \int_{T_{0}+u}^{T+u} w(t-u)\left|\xi\left(\sigma+i t, \omega_{1}\right)-\zeta_{n}\left(\sigma+i t, \omega_{1}\right)\right| d t \\
& =B \int_{-\infty}^{\infty}\left|l_{n}\left(\sigma_{2}-\sigma+i \tau\right)\right| \frac{1}{U} \int_{T_{0}+u+\tau}^{T+u+\tau} w(t-u-\tau)\left|\xi\left(\sigma_{2}+i t, \omega_{1}\right)\right| d t d \tau .
\end{aligned}
$$

By the Cauchy inequality we have

$$
\begin{aligned}
\int_{T_{0}+u+\tau}^{T+u+\tau} w & (t-u-\tau)\left|\xi\left(\sigma_{2}+i t, \omega_{1}\right)\right| d t \\
\leq & \left(\int_{T_{0}}^{T} w(t) d t \int_{T_{0}+u+\tau}^{T+u+\tau} w(t-u-\tau)\left|\xi\left(\sigma_{2}+i t, \omega_{1}\right)\right|^{2} d t\right)^{1 / 2} \\
& =\sqrt{U}\left(\int_{T_{0}+u+\tau}^{T+u+\tau} w(t-u-\tau)\left|\xi\left(\sigma_{2}+i t, \omega_{1}\right)\right|^{2} d t\right)^{1 / 2}
\end{aligned}
$$

Since $u$ is bounded, by Lemma 6 we have

$$
\int_{T_{0}+u+\tau}^{T+u+\tau} w(t-u-\tau)\left|\xi\left(\sigma_{2}+i t, \omega_{1}\right)\right|^{2} d t=B U(1+|\tau|)^{\alpha} .
$$

Hence and from (19) it follows that the left-hand side of the equality (18) is estimated by

$$
\begin{aligned}
B \sup _{\sigma+i u \in L} \int_{-\infty}^{\infty}\left|l_{n}\left(\sigma_{2}-\sigma+i \tau\right)\right|(1+|\tau|)^{\alpha} d \tau \\
=B \sup _{\sigma \in[-1 / 2,-\varepsilon / 4]} \int_{-\infty}^{\infty}\left|l_{n}(\sigma+i \tau)\right|(1+|\tau|)^{\alpha} d \tau .
\end{aligned}
$$

Moreover, from the definition of $l_{n}(s)$ we have

$$
\lim _{n \rightarrow \infty} \sup _{\sigma \in[-1 / 2,-\varepsilon / 4]} \int_{-\infty}^{\infty}\left|l_{n}(\sigma+i \tau)\right|(1+|\tau|)^{\alpha} d \tau=0 .
$$

This together with (20) and (17) proves the lemma. 
Let

$$
\widetilde{P}_{1, T, w}(A)=\frac{1}{U} \int_{T_{0}}^{T} w(\tau) I_{\left\{\tau: \xi\left(s+i \tau, \omega_{1}\right) \in A\right\}} d \tau, \quad A \in \mathcal{B}\left(H\left(D_{1}\right)\right) .
$$

Lemma 8. There exists a probability measure $P_{w}^{1}$ on $\left(H\left(D_{1}\right), \mathcal{B}\left(H\left(D_{1}\right)\right)\right)$ such that the measures $P_{1, T, w}$ and $\widetilde{P}_{1, T, w}$ both converge to $P_{w}^{1}$ as $T \rightarrow \infty$.

Proof. We will use the scheme of the proof of Lemma 4. By this lemma both the measures $P_{T, n, w}^{1}$ and $\widetilde{P}_{T, n, w}^{1}$ converge weakly to the same measure $P_{n, w}^{1}$ as $T \rightarrow \infty$. First we will prove that the family $\left\{P_{n, w}^{1}\right\}$ of probability measures is tight.

Let $\eta$ be a random variable defined as above, and let

$$
X_{T, n}(s)=\zeta_{n}(s+i \eta) .
$$

Then

$$
X_{T, n} \underset{T \rightarrow \infty}{\stackrel{\mathcal{D}}{\longrightarrow}} X_{n}
$$

where $X_{n}$ is an $H\left(D_{1}\right)$-valued random element having distribution $P_{n, w}^{1}$. Since the series for $\zeta_{n}(s)$ is absolutely convergent for $\sigma>1 / 2$, it follows that

$$
\sup _{n \geq 1} \limsup _{T \rightarrow \infty} \frac{1}{U} \int_{T_{0}}^{T} w(\tau) \sup _{s \in K_{l}}\left|\zeta_{n}(s+i \tau)\right| d \tau \leq R_{l}<\infty .
$$

Here $K_{l}$ are the compact sets used in the proof of Lemma 4. By Chebyshev inequality we have

$$
\mathbb{P}\left(\sup _{s \in K_{l}}\left|X_{T, n}(s)\right|>M_{l}\right) \leq \frac{1}{M_{l} U} \int_{T_{0}}^{T} w(\tau) \sup _{s \in K_{l}}\left|\zeta_{n}(s+i \tau)\right| d \tau .
$$

Consequently,

$$
\begin{aligned}
\limsup _{T \rightarrow \infty} \mathbb{P}\left(\sup _{s \in K_{l}}\left|X_{T, n}(s)\right|>M_{l}\right) & \\
\leq \frac{1}{M_{l}} & \sup \limsup _{n \geq 1} \frac{1}{U} \int_{T_{0}}^{T} w(\tau) \sup _{s \in K_{l}}\left|\zeta_{n}(s+i \tau)\right| d \tau .
\end{aligned}
$$

Now let $\varepsilon>0$ be an arbitrary number, and we take $M_{l}=R_{l} 2^{l} / \varepsilon$. Then from (22) and (23) we deduce that

$$
\limsup _{T \rightarrow \infty} \mathbb{P}\left(\sup _{s \in K_{l}}\left|X_{T, n}(s)\right|>M_{l}\right) \leq \varepsilon / 2^{l}
$$

for all $l \in \mathbb{N}$. Define a function $h: H\left(D_{1}\right) \rightarrow \mathbb{R}$ by the formula

$$
h(f)=\sup _{s \in K_{l}}|f(s)|, \quad f \in H\left(D_{1}\right) .
$$


Obviously, $h$ is continuous, and therefore in view of (21) and Theorem 5.1 of $[2]$,

$$
\sup _{s \in K_{l}}\left|X_{T, n}(s)\right| \frac{\mathcal{D}}{T \rightarrow \infty} \sup _{s \in K_{l}}\left|X_{n}(s)\right| .
$$

Hence and from (24) we find that

$$
\mathbb{P}\left(\sup _{s \in K_{l}}\left|X_{n}(s)\right|>M_{l}\right) \leq \varepsilon / 2^{l}
$$

for all $l \in \mathbb{N}$. Set

$$
H_{\varepsilon}=\left\{f \in H\left(D_{1}\right): \sup _{s \in K_{l}}|f(s)| \leq M_{l}, l \geq 1\right\} .
$$

Since $K$ is compact and $K \subset D_{1}$ implies $K \subseteq K_{l}$ for some $l$, the set $H_{\varepsilon}$ of functions analytic on $D_{1}$ is uniformly bounded on every compact $K \subset D_{1}$, and thus by Lemma 6 of [7] it is a compact subset of $H\left(D_{1}\right)$. Moreover, by $(25)$,

$$
\mathbb{P}\left(X_{n}(s) \in H_{\varepsilon}\right) \geq 1-\varepsilon
$$

for all $n \geq 1$ and, since $P_{n, w}^{1}$ is the distribution of $X_{n}$,

$$
P_{n, w}^{1}\left(H_{\varepsilon}\right) \geq 1-\varepsilon
$$

for all $n \geq 1$. So we have proved that the family $\left\{P_{n, w}^{1}\right\}$ of measures is tight. Hence and by the Prokhorov theorem it follows that $\left\{P_{n, w}^{1}\right\}$ is also relatively compact.

Applying the Chebyshev inequality once more and Lemma 3, we deduce that for every $\varepsilon>0$,

$$
\begin{aligned}
\lim _{n \rightarrow \infty} \limsup _{T \rightarrow \infty} \frac{1}{U} \int_{T_{0}}^{T} w(\tau) I_{\left\{\tau: \varrho\left(\zeta(s+i \tau), \zeta_{n}(s+i \tau)\right) \geq \varepsilon\right\}} d \tau \\
\quad \leq \lim _{n \rightarrow \infty} \limsup _{T \rightarrow \infty} \frac{1}{\varepsilon U} \int_{T_{0}}^{T} w(\tau) \varrho\left(\zeta(s+i \tau), \zeta_{n}(s+i \tau)\right) d \tau=0 .
\end{aligned}
$$

Set $X_{T}(s)=\zeta(s+i \eta)$. Then the relation $(26)$ can be written as

$$
\lim _{n \rightarrow \infty} \limsup _{T \rightarrow \infty} \mathbb{P}\left(\varrho\left(X_{T, n}(s), X_{T}(s)\right) \geq \varepsilon\right)=0 .
$$

Since the family $\left\{P_{n, w}^{1}\right\}$ is relatively compact, we can find a subsequence $\left\{P_{n^{\prime}, w}^{1}\right\}$ which converges weakly to $P_{w}^{1}$, say, as $n \rightarrow \infty$. Then

$$
X_{n^{\prime}} \underset{n^{\prime} \rightarrow \infty}{\stackrel{\mathcal{D}}{\longrightarrow}} P_{w}^{1} \text {. }
$$

Hence and from (27) and (21), using Theorem 4.2 of [2], we obtain

$$
X_{T} \underset{T \rightarrow \infty}{\stackrel{\mathcal{D}}{\longrightarrow}} P_{w}^{1}
$$

Therefore, there exists the measure $P_{w}^{1}$ such that the measure $P_{1, T, w}$ converges weakly to $P_{w}^{1}$ as $T \rightarrow \infty$. The relation (28) in addition shows that 
$P_{w}^{1}$ is independent of the choice of the subsequence $\left\{P_{n^{\prime}, w}^{\prime}\right\}$. Hence by Theorem 2.3 of $[2]$ we find that

$$
X_{n} \underset{n \rightarrow \infty}{\stackrel{\mathcal{D}}{\longrightarrow}} P_{w}^{1}
$$

Reasoning as above in the case of random elements

$$
\widetilde{X}_{T, n}\left(s, \omega_{1}\right)=\zeta_{n}\left(s+i \eta, \omega_{1}\right) \quad \text { and } \quad \widetilde{X}_{T}\left(s, \omega_{1}\right)=\xi\left(s+i \eta, \omega_{1}\right),
$$

and applying Lemma 7 and (29), we conclude that the measure $\widetilde{P}_{1, T, w}$ also converges weakly to $P_{w}^{1}$ as $T \rightarrow \infty$. The lemma is proved.

Proof of Theorem. As noted above, we consider the case $j=1$ only. Lemma 8 asserts that the measures $P_{1, T, w}$ and $\widetilde{P}_{1, T, w}$ converge weakly to some measure $P_{w}^{1}$ as $T \rightarrow \infty$ simultaneously. It remains to prove that $P_{w}^{1}=P_{1, \zeta}$. By the above remarks, it suffices to show that $P_{w}^{1}=P_{1, \xi}$, where $P_{1, \xi}$ denotes the distribution of $\xi(s, \omega)$ restricted to $\left(H\left(D_{1}\right), \mathcal{B}\left(H\left(D_{1}\right)\right)\right)$.

Let $A \in \mathcal{B}\left(H\left(D_{1}\right)\right)$ be a continuity set of $P_{w}^{1}$. Then the properties of weak convergence and Lemma 8 imply that

$$
\lim _{T \rightarrow \infty} \frac{1}{U} \int_{T_{0}}^{T} w(\tau) I_{\left\{\tau: \xi\left(s+i \tau, \omega_{1}\right) \in A\right\}} d \tau=P_{w}^{1}(A) .
$$

Let us fix $A$ and define the random variable $\theta$ on $(\Omega, \mathcal{B}(\Omega))$ by

$$
\theta(\omega)= \begin{cases}1 & \text { if } \xi(s, \omega) \in A \\ 0 & \text { if } \xi(s, \omega) \notin A\end{cases}
$$

It is easy to see that

$$
E(\theta)=\int_{\Omega} \theta d m=m(\omega: \xi(s, \omega) \in A)=P_{1, \xi}(A)<\infty .
$$

Taking into account Lemma 5 and reasoning similarly to the proof of Lemma 6 , we find that $\theta\left(\varphi_{\tau}(\omega)\right)$ is an ergodic process. Therefore, by (2) with $t=0$,

$$
\lim _{T \rightarrow \infty} \frac{1}{U} \int_{T_{0}}^{T} w(\tau) \theta\left(\varphi_{\tau}(\omega)\right) d \tau=E(\theta)
$$

for almost all $\omega \in \Omega$. But from the definitions of the random variable $\theta$ and of the one-parameter group, it follows that

$$
\begin{aligned}
\frac{1}{U} \int_{T_{0}}^{T} w(\tau) \theta\left(\varphi_{\tau}(\omega)\right) d \tau & =\frac{1}{U} \int_{T_{0}}^{T} w(\tau) I_{\left\{\tau: \xi\left(s, \varphi_{\tau}(\omega)\right) \in A\right\}} d \tau \\
& =\frac{1}{U} \int_{T_{0}}^{T} w(\tau) I_{\{\tau: \xi(s+i \tau, \omega) \in A\}} d \tau
\end{aligned}
$$


This, (31) and (32) give

$$
\lim _{T \rightarrow \infty} \frac{1}{U} \int_{T_{0}}^{T} w(\tau) I_{\{\tau: \xi(s+i \tau, \omega) \in A\}} d \tau=P_{1, \xi}(A)
$$

for almost all $\omega \in \Omega$. Thus, by (30),

$$
P_{w}^{1}(A)=P_{1, \xi}(A)
$$

for any continuity set of measure $P_{w}^{1}$. Since the continuity sets constitute the determining class, we have

$$
P_{w}^{1}(A)=P_{1, \xi}(A)
$$

for all $A \in \mathcal{B}\left(H\left(D_{1}\right)\right)$. The Theorem is proved.

The limit measure $P_{j, \zeta}$ in Theorem coincides with that when $w(t) \equiv 1$. This shows that the condition $(2)$ for $w(t)$ is sufficiently strong. It is of interest to find some condition for $w(t)$ that the relation (2) were satisfied. For example, (2) is true for $t=0$ if $w(t)$ is non-decreasing. But in this case the Theorem is a consequence of the similar theorem with $w(t) \equiv 1$ [5].

Now we give an example of a weight function $w(t)$ satisfying (2). Let $\mu(T)=\inf _{t \in\left[T_{0}, T\right]} w(t)$, and suppose that

$$
w(T) / \mu(T)=B .
$$

For simplicity we limit ourselves to ergodic processes with continuous sample paths. Let $\operatorname{EX}(0, \cdot)=A$. Then the Birkhoff-Khinchin theorem yields

$$
\int_{0}^{v} X(\tau, \omega) d \tau=v A+o(v)
$$

almost surely as $v \rightarrow \infty$. Hence we find that

$$
\int_{0}^{v} X(t+\tau, \omega) d \tau=v A+B|t|+o(v)=v A+v o(1+|t|)
$$

almost surely as $v \rightarrow \infty$. Integrating by parts we obtain

$$
\frac{1}{U} \int_{T_{0}}^{T} w(\tau) X(t+\tau, \omega) d \tau=\frac{1}{U} \int_{T_{0}}^{T} w(\tau)\left(d \int_{0}^{\tau} X(t+u, \omega) d u\right) d \tau .
$$

From (34) we have

$$
\int_{0}^{\tau} X(t+u, \omega) d u=\tau A+r(\tau) \tau(1+|t|),
$$

where $r(\tau) \rightarrow 0$ as $\tau \rightarrow \infty$. Consequently, since $U \geq \mu(T)\left(T-T_{0}\right)$, (35) 
gives in view of (33),

$$
\text { (36) } \begin{aligned}
\frac{1}{U} \int_{T_{0}}^{T} w(\tau) X(t+\tau, \omega) d \tau= & \frac{1}{U} w(T)(A T+r(T) T(1+|t|)) \\
& +\frac{B|t|}{U}-\frac{1}{U} \int_{T_{0}}^{T}(A \tau+r(\tau) \tau(1+|t|)) d w(\tau) \\
= & A+B(1+|t|) r(T)-\frac{1+|t|}{U} \int_{T_{0}}^{T} r(\tau) \tau d w(\tau) .
\end{aligned}
$$

Now let $T_{1} \rightarrow \infty$ as $T \rightarrow \infty$ and $T_{1}$ be such that

$$
\int_{T_{0}}^{T_{1}} r(\tau) \tau d w(\tau)=o(U), \quad T_{1} w\left(T_{1}\right)=o(U)
$$

as $T \rightarrow \infty$. Then again in view of (33),

$$
\begin{aligned}
\frac{1}{U} \int_{T_{0}}^{T} r(\tau) \tau d w(\tau) & =\frac{1}{U} \int_{T_{1}}^{T} r(\tau) \tau d w(\tau)+o(1) \\
& =\frac{B}{U} \sup _{\tau \in\left[T_{1}, T\right]}|r(\tau)| \int_{T_{1}}^{T} \tau d w(\tau)=o(1)
\end{aligned}
$$

as $T \rightarrow \infty$. Now (36) yields (2) with $\alpha=1$.

The latter note shows that the class of functions $w(t)$ in the Theorem is sufficiently wide.

The research described in this publication was made possible in part by Grant No. LAC000 from the International Science Foundation.

The authors express their gratitude to the referee for useful remarks.

\section{References}

[1] B. Bagchi, The statistical behaviour and universality properties of the Riemann zeta function and other allied Dirichlet series, Ph.D. thesis, Indian Statistical Institute, Calcutta, 1981.

[2] P. Billingsley, Convergence of Probability Measures, Wiley, New York, 1968.

[3] H. Cramér and M. R. Leadbetter, Stationary and Related Stochastic Processes, Wiley, New York, 1967.

[4] A. Laurinčikas, On limit theorems for the Riemann zeta function in some spaces, in: Probab. Theory and Math. Statistics, Proceeding of Sixth Vilnius Intern. Conf., B. Grigelionis et al. (eds.), VSP/TEV, 1994, 457-483. 
[5] A. Laurinčikas, Limit theorems for a product of the Hurwitz zeta-function, Liet. Mat. Rink. 34 (1994), 197-210 (in Russian).

[6] —, Limit Theorems for the Riemann Zeta-Function, Kluwer, 1996.

[7] A. Laurinčikas and G. Misevičius, A limit theorem with weight for the Riemann zeta-function in the space of analytic functions, Liet. Mat. Rink. 34 (1994), 211-224 (in Russian).

Department of Mathematics

Vilnius University

Naugarduko, 24

2006 Vilnius, Lithuania

E-mail: antanas.laurincikas@maf.vu.lt

Received on 8.12.1994

and in revised form on 4.3 .1996 